\title{
Oxaliplatin-induced acute-onset thrombocytopenia and hemorrhage: Case report and review of the literature
}

\author{
SHOICHIRO OHTA ${ }^{1,3}$, YUKIKO CHO ${ }^{1}$, SHINJI OSHIMA ${ }^{2}$, OSAMU HOSOYA ${ }^{2}$, \\ KAZUHIKO JUNI ${ }^{2}$ and HIROSHI KOJIMA ${ }^{1}$ \\ ${ }^{1}$ Department of Oncology, Ibaraki Prefectural Central Hospital, Ibaraki; ${ }^{2}$ Laboratory of Pharmacology, \\ Faculty of Pharmaceutical Sciences, Josai University, Saitama, Japan
}

Received December 13, 2011; Accepted March 19, 2012

DOI: $10.3892 / \mathrm{ol} .2012 .653$

\begin{abstract}
We report the case of a woman who developed acute thrombocytopenia with hemorrhagic diathesis during adjuvant treatment of colorectal adenocarcinoma with oxaliplatin, 5-fluorouracil and leucovorin. A 55-year-old woman started adjuvant chemotherapy with oxaliplatin, 5-fluorouracil and leucovorin (mFOLFOX6). Prior to starting the 12th course of chemotherapy, a complete blood cell count showed the following values: neutrophils $1800 / \mathrm{mm}^{3}$, platelets $136,000 / \mathrm{m}^{3}$ and hemoglobin $11.1 \mathrm{~g} / \mathrm{dl}$. A blood count revealed that the platelet levels had dropped to $35,000 / \mathrm{mm}^{3}$, with no significant changes in hemoglobin levels following the course. The administration of corticosteroids was begun and the platelet number was recovered. Clinicians should be aware of the possibility of oxaliplatin-induced hematological emergencies during the treatment of colorectal cancer patients in order to optimize supportive treatment and avoid toxic mortality.
\end{abstract}

\section{Introduction}

Oxaliplatin is a third-generation platinum derivative that has been widely used in patients with gastrointestinal malignancies, including colorectal cancer (CRC). Results of a number of studies showed that the combination of 5-fluorouracil, leucovorin and oxaliplatin (FOLFOX) increased survival rate and reduced the risk of disease progression in patients with metastatic CRC and stage III colon cancer $(1,2)$. Thrombocytopenia has been noted in more than $70 \%$ of patients receiving FOLFOX, but is usually self-limited and related to myelosuppression from oxaliplatin $(3,4)$. The isolated and acute decline in platelets after FOLFOX treat-

Correspondence to: Dr Shoichiro Ohta, Department of Oncology, Ibaraki Prefectural Central Hospital, 6528 Koibuchi, Kasama, 309-1793, Japan

E-mail: d11494@hotmail.co.jp

Present address: ${ }^{3}$ Kan-Etsu Hospital, 145-1 Suneori, Tsurugashima, Japan

Key words: oxaliplatin, thrombocytopenia, mFOLFOX6 ment is thought to be immune-mediated, and is referred to as drug-induced immune thrombocytopenia (DIIT). Oxaliplatindependent antibody against the platelet glycoprotein IIb/IIIa complex has been identified in patients with oxaliplatininduced immune thrombocytopenia (5).

Drug-induced acute thrombocytopenia is an extremely rare side effect that may occur immediately after oxaliplatin infusion $(3,4)$. This potentially fatal reaction is immune-mediated and may be anticipated based on mild hemorrhagic signs during previous administration. This is the first report of acute thrombocytopenia occurring during adjuvant treatment of colorectal cancer with oxaliplatin. Clinicians should be aware of this adverse event to prevent possible serious consequences and stop further oxaliplatin administration. Oxaliplatin is a third-generation platinum agent that is active in the treatment of colorectal adenocarcinoma. Drug-induced immune thrombocytopenia and hemolytic anemia have been reported following the administration of several compounds including cytotoxic agents and platinum derivatives. Oxaliplatin is capable of inducing the formation of drug-dependent antibodies that react with red blood cells or, less frequently, thrombocytes. In the presence of sensitizing plasma concentrations of oxaliplatin, these antibodies are able to mediate the peripheral destruction of blood cells, leading to acute hemolytic anemia, thrombocytopenia, or both (Evan's syndrome) (5). We report the case of a woman who developed acute thrombocytopenia with hemorrhagic diathesis during adjuvant treatment of colorectal adenocarcinoma with oxaliplatin, 5-fluorouracil and leucovorin. The study was aprroved by the ethics committee of the university, and patient consent was obtained.

\section{Case report}

A 55-year-old woman without relevant comorbidities underwent sigmoidectomy in August 2007 for stage III colon adenocarcinoma. In September 2007, the patient started adjuvant chemotherapy with oxaliplatin, 5-fluorouracil and leucovorin (FOLFOX-4), which caused the development of grade 3 neurotoxicity after the 11 th administration. Prior to starting the last (12th) course of chemotherapy, a complete blood cell count showed the following values: neutrophils $1800 / \mathrm{mm}^{3}$, platelets $136,000 / \mathrm{mm}^{3}$, and hemoglobin $11.1 \mathrm{~g} / \mathrm{dl}$. A blood count revealed that the platelet level had dropped to 
Table I. Review of 20 cases of oxaliplatin-induced acute-onset thrombocytopenia and hemorrhage.

\begin{tabular}{|c|c|c|c|c|c|c|c|c|c|}
\hline Case & Gender & Age & Course & $\begin{array}{l}\text { Platelet } \\
\text { count }\end{array}$ & $\begin{array}{l}\text { Platelet } \\
\text { (after) }\end{array}$ & Onset & Symptoms & Treatment & Result \\
\hline 1 & M & 56 & $\mathrm{~N} / \mathrm{K}$ & 99000 & 6000 & $5 \mathrm{~h}$ & $\begin{array}{l}\text { Purpura, } \\
\text { gingivorrhagia }\end{array}$ & Steroids, transfusion & Recovery \\
\hline 2 & M & 52 & 9 & 182000 & 17000 & 1 day & Bleeding & Transfusion & Recovery \\
\hline 3 & $\mathrm{~F}$ & 40 & 13 & 153000 & 80000 & 1 day & Back pain & No & Recovery \\
\hline 4 & $\mathrm{~F}$ & 70 & 19 & 432000 & 2000 & $4 \mathrm{~h}$ & Skin rash & Transfusion & Recovery \\
\hline 5 & M & 60 & 14 & 143000 & 2000 & $1 \mathrm{~h}$ & Hematemesis & Transfusion & Recovery \\
\hline 6 & $\mathrm{~F}$ & 79 & 15 & 221000 & 5000 & $4 \mathrm{~h}$ & Bleeding, purpura & Steroids, transfusion & Recovery \\
\hline 7 & $\mathrm{~F}$ & 38 & 17 & 150000 & 13000 & 2 days & $\begin{array}{l}\text { Abdominal pain, } \\
\text { petechial hemorrhages }\end{array}$ & Transfusion & Recovery \\
\hline 8 & $\mathrm{~F}$ & 55 & 10 & 136000 & 6000 & $24 \mathrm{~h}$ & Not mentioned & Transfusion & Recovery \\
\hline 9 & $\mathrm{~F}$ & 59 & 20 & 253000 & 5000 & $8 \mathrm{~h}$ & Purpura, gingivorrhagia & Steroids, transfusion & Recovery \\
\hline 10 & M & 64 & 24 & 163000 & 4000 & $1 \mathrm{~h}$ & Back pain & Transfusion & Death \\
\hline 11 & $\mathrm{~F}$ & 57 & 11 & 89000 & 17000 & $17 \mathrm{~h}$ & Bleeding & Transfusion & Recovery \\
\hline 12 & $\mathrm{~F}$ & 50 & 15 & 119000 & 5000 & $7 \mathrm{~h}$ & Purpura, gingivorrhagia & Steroids, transfusion & Recovery \\
\hline 13 & M & 48 & 21 & 94000 & 1000 & $7 \mathrm{~h}$ & Purpura & Steroids, transfusion & Recovery \\
\hline 14 & $\mathrm{~F}$ & 59 & 12 & 151000 & 20000 & 1 day & Petechial hemorrhages & Steroids, transfusion & Recovery \\
\hline 15 & M & 60 & 3 & 226000 & 4000 & 1 day & $\begin{array}{l}\text { Petechial hemorrhages, } \\
\text { tongue hematoma }\end{array}$ & IVIG infusion & Recovery \\
\hline 16 & $\mathrm{~F}$ & 66 & 3 & 87000 & 66000 & $5-10 \min$ & Skin rash & Steroids & Recovery \\
\hline 17 & $\mathrm{~F}$ & 55 & 13 & 136000 & 8000 & Immediately & Petechial hemorrhages & Steroids & Recovery \\
\hline 18 & M & 78 & 17 & 104000 & 15000 & $1.5 \mathrm{~h}$ & Coma & $\begin{array}{l}\text { Steroids, transfusion, } \\
\text { G-CSF }\end{array}$ & Death \\
\hline 19 & $\mathrm{~F}$ & 66 & 25 & $\mathrm{~N} / \mathrm{K}$ & 22000 & 4 days & $\begin{array}{l}\text { Fever up, general } \\
\text { fatigue }\end{array}$ & Transfusion, HD & Recovery \\
\hline $\begin{array}{l}\text { Our } \\
\text { case }\end{array}$ & $\mathrm{F}$ & 55 & 9 & 135000 & 35000 & 2 days & Bleeding & Steroids, transfusion & Recovery \\
\hline
\end{tabular}

NK, not known; G-CSF, granulocyte colony-stimulating factor; HD, hemodialysis; IVIG, intravenous immunoglobulin.

\section{Platelet count $\left(/ \mathrm{mm}^{3}\right)$}

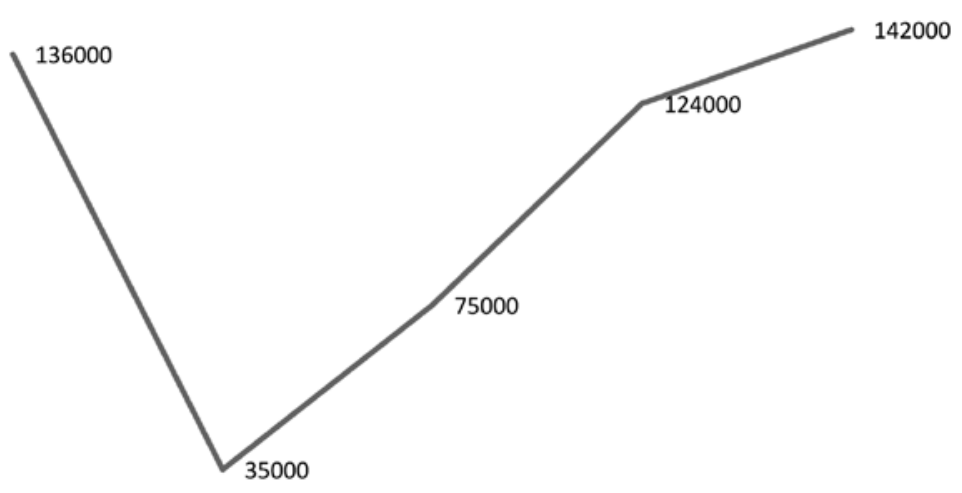

$\begin{array}{llll}\text { Pre-treatment } & \text { onset } & 1 \text { day } & 4 \text { days }\end{array}$


$35,000 / \mathrm{mm}^{3}$, with no significant changes in the hemoglobin level. The D-dimer value increased to $1.2 \mathrm{mg} / \mathrm{ml}$. The direct antiglobulin test (DAT) was strongly positive, with slight increases in lactate dehydrogenase $(\mathrm{LDH}=664 \mathrm{U} / 1)$ and reticulocytes $(2.9 \%)$, whereas no relevant abnormalities in bilirubin, haptoglobin or complement factors (C3 and $\mathrm{C} 4)$ were observed. The administration of corticosteroids was begun. Since the blood cell count returned to acceptable levels within four days (Fig. 1), bone marrow biopsy was not performed. The patient was subsequently discharged and laboratory test results remained in the normal range during follow-up.

\section{Discussion}

Patients treated with oxaliplatin may develop allergic or idiosyncratic reactions and, rarely, hematological emergencies (3-5). Few cases of immune-mediated hemolytic anemia and/or thrombocytopenia due to repeated oxaliplatin infusions have been reported in the literature. To the best of our knowledge, there are 20 reports of oxaliplatin-mediated acute thrombocytopenia during adjuvant treatment of colorectal cancer, whereas several occurrences have been described in the metastatic setting (3-13). Anemia and thrombocytopenia due to bone marrow suppression are often observed during treatment with platinum compounds (cisplatin, carboplatin or oxaliplatin). Nevertheless, acute-onset hemolytic anemia rarely occurs immediately after the administration of platinum salts, including oxaliplatin (9). The mechanism of hemolysis is immune-mediated, but remains controversial and is not completely understood (10). Some authors described a penicillin-like reaction due to the absorption of oxaliplatin into the cell membrane of erythrocytes, with consequent exposure of strongly immunogenic epitopes of surface glycoproteins (11). This mechanism leads to the formation of drug-dependent antibodies that mediate the peripheral destruction of erythrocytes in the presence of the sensitizing compound. It is likely that the repeated administration of oxaliplatin is necessary to induce and maintain the immune-mediated response in sensitized subjects (3). All the known case reports share a high cumulative dose of oxaliplatin administered prior to the appearance of the immune reaction (3). Hemolysis may be associated with fever, chills and abdominal or back pain, mimicking an allergic reaction pattern, which is occasionally observed during oxaliplatin infusion. Corticosteroid premedication suppresses the immune-mediated mechanism, contributing to the underestimation of oxaliplatin-induced acute hematological disorders (10).

We investigated 20 cases including that of the present study (3-13). There were 7 male cases and 13 female cases (Table I). The patients ranged in age from 38 to 79 years old, with an average of 58 years of age. Acute thrombocytopenia occurred between the 3rd course and 24th course of L-OHP administration, with the average number of courses being 14.2. There were 14 cases in which symptoms were observed during and after the 10th course. This result indicates that the symptoms are more likely to develop with an increase in the number of administrations. The symptoms were observed in the $12 \mathrm{~h}$ after administration in 11 cases $(55 \%)$ and from 12 to $24 \mathrm{~h}$ after administration in 2 cases (10\%), with both of these time-frames representing precipitous onset. As an initial symptom, bleeding from a purple spot or port-site due to thrombocytopenia was observed in many cases $(60 \%)$.

A recommended treatment for drug-induced thrombocytopenia is to stop ingesting the drugs that are presumed to be the cause. However, for serious thrombocytopenia associated with mucosal bleeding, aggressive treatment is preferable (12). This type of treatment might include platelet transfusion, adrenocortical steroid hormone administration, hormone intravenous high-dose $\gamma$-globulin therapy or plasma exchange therapy (Table I). For case 4, since the platelet count was at an abnormally low level of $0.1 \times 10 \mu 1,10$ units of platelet transfusion and steroid pulse were carried out (Table I).

In terms of prognosis, 12 cases showed improvement, but one case in which cerebral hernia developed due to cerebral hemorrhage resulted in death six days later (Table I). In addition, in two cases, the same regimen was carried out after improvement was noted prior to thrombocytopenia occurring again. However, it was concluded that the regimen needed to be changed if thrombocytopenia developed. A sudden decrease in platelet levels, if not promptly recognized and treated, may be life-threatening due to the risk of acute hemorrhage. Furthermore, an immunohemolytic reaction may threaten the life of the patient because of the possibility of severe anemia and/or acute renal failure.

Female patients with advanced CRC and prior oxaliplatin exposure are more likely to develop this consequence, although it may also occur in male patients. Therefore, it is imperative that patients be thoroughly examined for signs and symptoms of bleeding with concurrent complete blood count evaluation even after recovery from hypersensitivity symptoms. Heightened vigilance and prompt testing may be useful in recognizing the development of antibodies in patients with religious objections to transfusion prior to the onset of severe thrombocytopenia. Following the discontinuation of oxaliplatin, patients often undergo concurrent platelet transfusion, if indicated. Additional tests such as a peripheral blood smear examination and direct antiglobulin test are also useful in the assessment for Evan's syndrome or hemolytic-uremic syndrome in the case of worsening anemia or hemolysis. Patients with documented oxaliplatin-induced acute thrombocytopenia should not be re-challenged with oxaliplatin and should have oxaliplatin listed as a drug to which they have an allergy.

In conclusion, clinicians should be aware of the possibility of oxaliplatin-induced hematological emergencies during the treatment of CRC patients to optimize supportive treatment and avoid toxic mortality. Signs or symptoms such as epistaxis, even if mild, should be taken into account during treatment with oxaliplatin, as they may signal the subsequent development of serious adverse events.

\section{References}

1. Laurie JA, Moertel CG, Fleming TR, et al: Surgical adjuvant therapy of large-bowel carcinoma: an evaluation of levamisole and the combination of levamisole and fluorouracil. The North Central Cancer Treatment Group and the Mayo Clinic. J Clin Oncol 7: 1447-1456, 1989.

2. Kanemitsu Y, Kato T, Shimizu Y, et al: A randomized phase II/ III trial comparing hepatectomy followed by mFOLFOX6 with hepatectomy alone as treatment for liver metastasis from colorectal cancer: Japan Clinical Oncology Group Study JCOG0603. Jpn J Clin Oncol 39: 406-409, 2009. 
3. Polyzos A, Tsavaris N, Gogas H, et al: Clinical features of hypersensitivity reactions to oxaliplatin: a 10 -year experience. Oncology 76: 36-41, 2008.

4. Earle CC, Chen WY, Ryan DP and Mayer RJ: Oxaliplatininduced Evan's syndrome. Br J Cancer 84: 441, 2001.

5. Sorbye H, Bruserud O and Dahl O: Oxaliplatin-induced haematological emergency with an immediate severe thrombocytopenia and haemolysis. Acta Oncol 40: 882-883, 2001.

6. Yasuhara E, Nitta Y, Yasuda K, Maruyama M and Akazai Y: Two cases of Oxaliplatin-induced thrombocytopenia. Jpn J Clin Surg 71: 913-917, 2010

7. Pietrantonio F, Di Bartolomeo M, Buzzoni R and Bajetta E: Acute immune-mediated thrombocytopenia due to oxaliplatin administration: a case report. Tumori 96: 154-156, 2010.

8. Bautista MA, Stevens WT, Chen CS, Curtis BR, Aster RH and Hsueh CT: Hypersensitivity reaction and acute immunemediated thrombocytopenia from oxaliplatin: two case reports and a review of the literature. J Hematol Oncol 26: 3-12, 2010.
9. Shao YY and Hong RL: Fatal thrombocytopenia after oxaliplatin-based chemotherapy. Anticancer Res 28: 3115-3118, 2008.

10. Taleghani BM, Meyer O, Fontana S, et al: Oxaliplatin-induced immune pancytopenia. Transfusion 45: 704-708, 2005.

11. Curtis BR, Kaliszewski J, Marques MB, et al: Immune-mediated thrombocytopenia resulting from sensitivity to oxaliplatin. Am J Hematol 81: 199-201, 2006.

12. Dold FG and Mitchell EP: Sudden-onset thrombocytopenia with oxaliplatin. Ann Intern Med 139: 156-157, 2003.

13. Koutras AK, Makatsoris M, Paliogianni F, et al: Oxaliplatininduced acute-onset thrombocytopenia, hemorrhage and hemolysis. Oncology 67: 179-182, 2004. 